\title{
TEM studies of TbNiAl in the Disproportionation Stage of the HDDR Process
}

\author{
C.M. Andrei*, J. Walmsley ${ }^{\dagger}$, Y.D. Yu **, H.W. Brinks ${ }^{\dagger \dagger}$, R. Holmestad*, and B.C. Hauback ${ }^{\dagger \dagger}$
}

*Dept. of Physics, ${ }^{* *}$ Dept. of Chemistry, Norwegian University of Science and Technology, N-7491

Trondheim, Norway

${ }^{\dagger}$ SINTEF Materials Technology Applied Physics, 7465 Trondheim, Norway

${ }^{\dagger}$ Institute for Energy Technology, P.O. Box 40, N-2007 Kjeller, Norway

The hydrogenation disproportionation desorption recombination (HDDR) process is an effective way to obtain highly coercive magnets by refining originally coarse grains to fine grains $[1,2]$. The intermetallic compound $\mathrm{TbNiAl}$ can absorb up to 1.4 deuterium atoms per formula unit at room temperature and 1 bar $\mathrm{H}_{2} / \mathrm{D}_{2}$ pressure $[3,4]$. In the present work we have investigated the microstructural transformations during the disproportionation stage of the HDDR process in $\mathrm{TbNiAl}$ by transmission electron microscopy (TEM).

TbNiAl was prepared from $\mathrm{Tb}$ (purity 99,8\%), $\mathrm{Ni}(99,9 \%)$ and $\mathrm{Al}(99,9 \%)$ by arc melting in an argon atmosphere. The ingots were remelted several times to increase their homogenity and annealed at $800^{\circ} \mathrm{C}$. The material was first heated to $600^{\circ} \mathrm{C}$ in vacuum and the atmosphere was switched to 4 bar $\mathrm{D}_{2}$. X-ray diffraction (XRD) showed that the sample was disproportionated into two phases, $\mathrm{TbD}_{2.2}$ and NiAl. This is the so-called solid-HD(DR) experiment since the lumps of the starting material are maintained. TEM samples were prepared by mechanical grinding in a silicate crucible and spreading the dry powder on a holy carbon film supported on a titanium grid. Electron microscops was performed in a Philips CM 30 operated at $300 \mathrm{kV}$.

A dark field micrograph from the sample is shown in FIG 1a. This indicates that the structure consists of nanocrystalline regions with average sizes down to a few nanometers. Selected area diffraction shows the existence of both phases, the measured d spacing closely matching those of the $\mathrm{TbD}_{2.2}$ and $\mathrm{NiAl}$ phases from the XRD data. Some texture is evident in the diffraction pattern. For example, the third ring marked in FIG 1b, corresponding to the NiAl phase (110), is not continuous. The other rings, which are more continuous, correspond to the $\mathrm{TbD}_{2.2}$ phase. One method for studying the distribution of the phases in the disproportionated state is lattice resolution imaging, FIG 2. The detailed structure is shown in FIG 3 in which both the $\mathrm{TbD}_{2.2}$ and NiAl phases are present. The dominant $\mathrm{TbD}_{2.2}$ phase has the measured lattice spacing $\mathrm{d}_{112}=3.14 \AA$. Using the $\mathrm{TbD}_{2.2}$ as internal reference we measured a particle region with a lattice spacing of $2.04 \AA$ which corresponds well with the NiAl phase (110) spacing [5].

\section{References}

[1] T. Takeshita, R. Nakayama, Proc. of $10^{\text {th }}$ Int. Workshop on Re Magnets and Their Applications, 1989, 551.

[2] I.R. Harris, Proc. of $12^{\text {th }}$ Int. Workshop on Re Magnets and Their Applications, 1992, 347

[3] B.C. Hauback et al., J. Alloys and Compounds 293-295 (1999) 178-184

[4] H.W. Brinks et al., J. Alloys and Compounds 330 (2002) 169

[5] The authors thank Norwegian Research Council for financial support. 

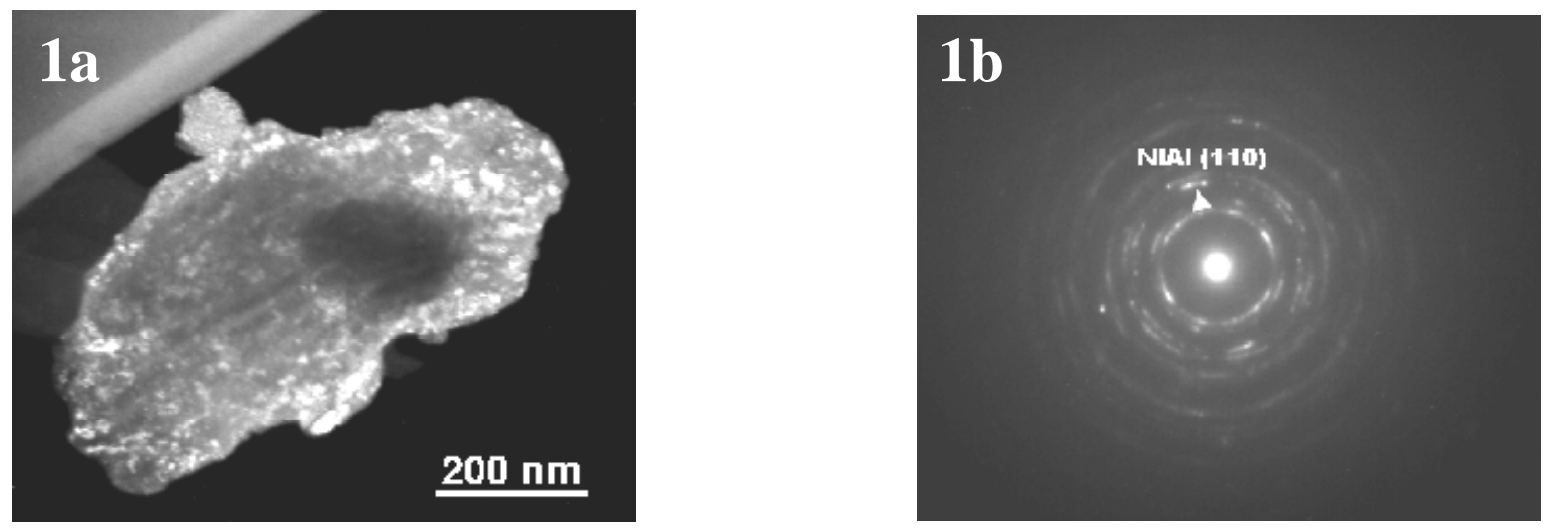

FIG 1a TEM dark field image and $1 \mathrm{~b}$ selected area diffraction pattern of the TbNiAl disproportionated state
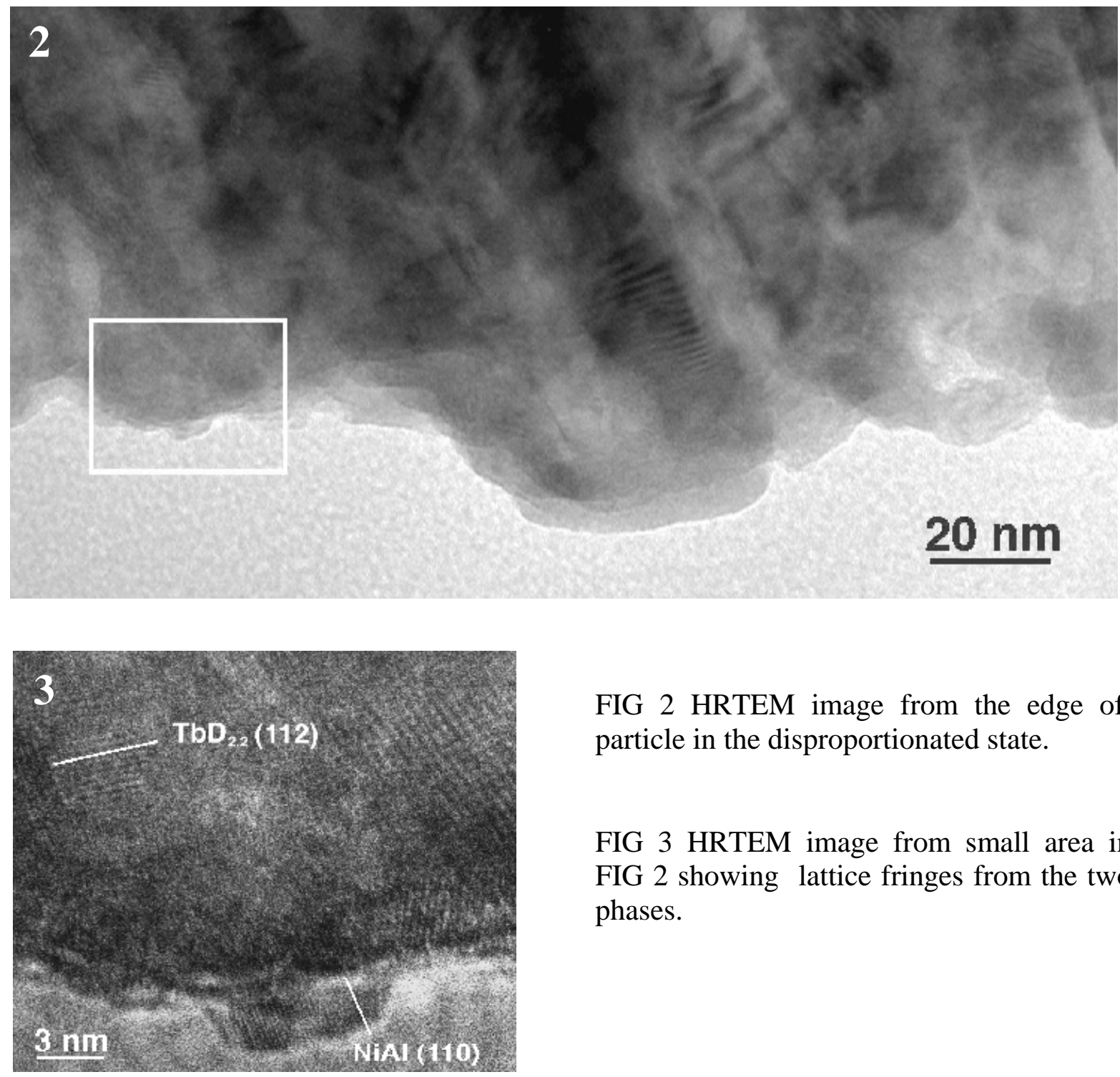

FIG 2 HRTEM image from the edge of a particle in the disproportionated state.

FIG 3 HRTEM image from small area in FIG 2 showing lattice fringes from the two phases. 